\title{
Evaluación de escala visual como medida de calidad interna y frescura de huevo comercial
}

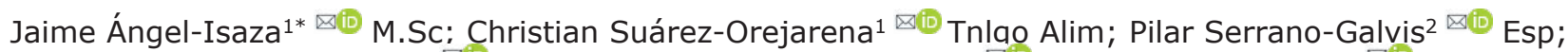

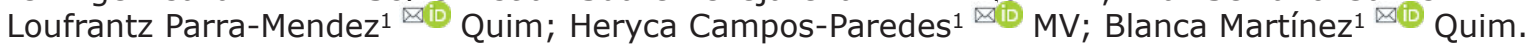

*Correspondencia: Nutricionanimal@promitec.com.co

Recibido: Mayo 2020; Aceptado: Noviembre 2020; Publicado: Marzo 2021.

\section{RESUMEN}

Objetivo. El objetivo del presente estudio fue evaluar el uso de una escala de valoración visual de calidad interna de huevo comercial y su relación con otras variables de calidad durante el almacenamiento. Materiales y métodos. Los análisis se realizaron en Laboratorio de Calidad de Huevo de Promitec Santander, se utilizaron 527 huevos comerciales de cinco regiones de Colombia. Los huevos se almacenaron entre 28 y $30^{\circ} \mathrm{C}$. Los días 3, 6, 8, 12 y 17 de almacenamiento se realizaron registros fotográficos de vistas frontal y superior para la puntuación de escala visual y se llevaron a cabo mediciones fisicoquímicas. Se utilizaron pruebas de ANOVA y TUKEY para comparar medias de cada variable entre los diferentes tiempos de medición. Las correlaciones entre las variables de calidad interna se analizaron mediante coeficientes de correlación además de regresión lineal. Resultados. valores de $\mathrm{pH}$ de la albumina y la yema presentaron un aumento significativo $(p<0.05)$ durante el tiempo de almacenamiento; por el contrario, el índice de yema decreció $(p<0.05)$ en el tiempo. Se observó correlación negativa entre el $\mathrm{pH}$ de la albumina con las variables de índice de yema y la escala visual. Por su parte la correlación entre el índice de yema y la escala visual fue positiva $(p<0.05)$. Conclusiones. El tiempo de almacenamiento a temperatura ambiente afecta las variables fisicoquímicas medidas de calidad, además disminuye la puntuación en la escala de evaluación visual; la cual permitió identificar y puntuar cambios físicos químicos observables en los componentes internos del huevo.

Palabras clave: Condiciones de almacenamiento; deterioro del huevo; gallinas de postura; propiedades del huevo (Fuente: Agrovoc, CAB).

\section{ABSTRACT}

Objective. We aimed to evaluate the use of visual assessment scale of internal egg quality and its relationship with other quality variables during storage time. Materials and methods. The analyses were carried out in the Egg Quality Laboratory of Promitec Santander. To the study, 527 commercial table eggs from five regions of Colombia were used. Eggs were stored between 28 and 
$30^{\circ} \mathrm{C}$. On days $3,6,8,12$, and 17 of storage, front and top view photographic records were made for visual scale scoring, and physicochemical measurements were performed. ANOVA and TUKEY test were used to compare means for each variable between the different measurement times. The correlations between the internal quality variables were analysed using correlation coefficient and linear regression. Results. Albumin and yolk pH increased and the visual scale and the yolk index decreased through the storage time $(p>0.05)$. A negative correlation was observed between the $\mathrm{pH}$ of albumin with the yolk index and the visual scale. On the other hand, a positive correlation between the yolk index and the visual scale was found $(p<0.05)$. Conclusions. Storage time at room temperature affects the physicochemical variables measured for quality, and also decreases the score on the visual evaluation scale; which allowed to identify and score observable physical chemical changes in the internal components of the egg.

Keywords: Egg deterioration; egg properties; layer chickens; storage conditions (Sources: Agrovoc, $C A B)$.

\section{INTRODUCCION}

En Colombia, la comercialización de huevo se ha intentado diferenciar en el mercado, apelando a la calidad y a la frescura como atributo diferenciador entre marcas. En la industria de la gallina ponedora comercial, es fundamental la producción de huevos que presenten características de buena calidad tanto externas como internas para disminuir pérdidas por descarte y además de lograr obtener huevos más frescos y de calidad hasta el consumidor final, el cual cada vez es más exigente con la frescura de los alimentos que llegan a su mesa (1).

En la vida útil del huevo, el trascurso de tiempo desde la postura del huevo hasta que llega al consumidor final, se presentan cambios fisicoquímicos que pueden modificar el sabor, la textura y en general la palatabilidad. Estos cambios son generados por una serie de reacciones químicas relacionadas con la transferencia de dióxido de carbono a través de la cáscara en la que la albumina densa se vuelve líquida, involucrando ácido carbónico $\left(\mathrm{H}_{2} \mathrm{CO}_{3}\right)$ como uno de los componentes del sistema de amortiguación de la albúmina, que se disocia en agua y dióxido de carbono $\left(\mathrm{CO}_{2}\right)$ alterándose el contenido total de sólidos y el pH (2).

Existen diversos indicadores de calidad interna y frescura del huevo, algunos asociados a la clara como son las unidades Hauhg (UH) y otros a la yema como el índice y altura de yema, además de variables dependientes del tiempo y la velocidad de intercambio de oxígeno y $\mathrm{CO}_{2}$ como el $\mathrm{pH}$, que permiten evaluar el grado de perdida de las características de calidad que presentan los componentes internos del huevo y por ende su frescura $(3,4)$. No obstante, su aplicación a nivel comercial o de los consumidores, no es fácil de implementar, por lo que se deben desarrollar métodos de evaluación de calidad y frescura que puedan ser utilizados de forma rápida y fácil por cualquier eslabón de la cadena productiva del huevo comercial. El objetivo del presente estudio fue evaluar el uso de una escala de valoración visual de calidad interna de huevo de gallina ponedora comercial y su relación con otras variables de calidad durante el tiempo de almacenamiento a temperatura ambiente.

\section{MATERIALES Y MÉTODOS}

Características del estudio. El presente estudio fue de tipo observacional retrospectivo. Para este se incluyó, un total de 527 huevos (blanco y marrón) de gallinas ponedoras comerciales con peso promedio de $60 \pm 6.2 \mathrm{~g}$, provenientes de granjas productivas ubicadas en los departamentos del Valle del Cauca, Cauca, Antioquia, Caldas, Cundinamarca y Santander en Colombia. Los huevos fueron transportados el mismo día de la recolección en granja, empacados en cubetas plásticas de 30 unidades utilizando cavas de poliestireno expandido como medio de protección. Los huevos fueron recibidos y analizados en el Laboratorio de Calidad de Huevo de la empresa Promitec Santander entre las fechas del 29 de marzo de 2019 y el 14 de enero de 2020.

Las muestras procedentes de diferentes empresas productoras de huevo comercial fueron tomadas en las horas de la tarde una vez habían sido clasificados en los respectivos centros de distribución y remitidos al laboratorio de tal manera que a su llegada no sobrepasaran los 3 días de postura. Una vez recibidas las muestras se realizó inspección visual y ovoscópica para integridad de la cascara descartando aquellos con 
roturas macroscópicas. Los huevos seleccionados como viables para ser analizados se almacenaron en bandejas plásticas por 30 unidades a temperatura ambiente que osciló entre 28 y $30^{\circ} \mathrm{C}$ con humedad relativa entre 58 a $64 \%$.

El análisis de calidad interna de los huevos se llevó a cabo los días 3,6, 8,12 y 17 de almacenamiento teniendo como día cero el día de la postura del huevo. Para el análisis, en cada uno de los días establecidos se tomaron cuatro huevos de manera aleatoria de cada bandeja; cada unidad fue pesada utilizando una balanza digital con precisión de dos decimales antes de retirar su cáscara para posteriormente depositar el contenido sobre un recipiente de acetato transparente de $11,5 \mathrm{~cm}$ de diámetro y $1 \mathrm{~cm}$ de profundidad. Finalmente, los componentes de cada huevo (cáscara, albumen y yema) fueron separados mecánicamente y pesados de forma individual, estos pesos se expresaron como peso absoluto y peso relativo que indica el porcentaje que representa cada componente del peso total del huevo además mediante la división entre el peso relativo de la yema y la clara se obtuvo la relación yema: albumen.

pH de albúmina y yema. Con los componentes internos del huevo ya separados; se llevó a cabo la a medición del $\mathrm{pH}$ de cada una de las fracciones internas utilizando un medidor digital de $\mathrm{pH}$ marca Lutron ${ }^{\circledR} \mathrm{PH}-207 \mathrm{HA}$.

Índice de yema (IY). Utilizando una tableta mediaPad M3 con resolución de 8 megapíxeles, se realizaron dos registros fotográficos: uno frontal $(6 \mathrm{~cm}$ de distancia entre la cámara y el huevo), y uno superior ( $23 \mathrm{~cm}$ entre la cámara y el huevo) (Figura 1). Utilizando las dos fotografías y mediante el software libre de procesamiento de imagen Image ${ }^{\circledR}$ se realizó la medición en milímetros de la altura de la yema y el diámetro de yema. Con las mediciones referentes a la yema se empleó la fórmula:

$\mathrm{IY}=($ Altura de yema $(\mathrm{mm})) /($ Diametro de yema $(\mathrm{mm}))$

Escala visual de calidad interna de huevo. La escala de evaluación visual de calidad interna de huevo empleada en la investigación se desarrolló con base a la modificación de en las escalas de medición de frescura del Departamento de Agricultura de los Estados Unidos (USDA) referenciado por Sastre (5) en 2002 y la escala reportada por Van y Wilgus (6) en 1935.

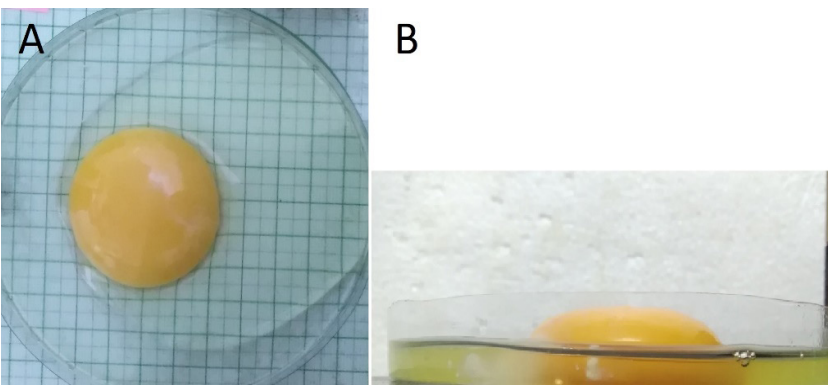

Figura 1. Vista superior (A) y frontal (B) utilizadas para la determinación del índice de yema y la puntuación de la escala de evaluación de calidad interna de huevo.

Para la puntuación de los huevos en la escala de evaluación visual se utilizaron dos fotografías de cada huevo, una con la vista frontal y otra desde la vista superior (Figura 1) las cuales fueron evaluadas por tres personas del laboratorio de calidad de huevo de forma independiente. Cada una de las dos vistas se evaluaron y puntuaron por separado dentro de la escala, obteniendo un valor de puntuación frontal y uno superior. Posteriormente los dos valores de cada huevo (la puntuación frontal y la puntuación superior) fueron promediados para obtener una puntuación de escala visual promedio para cada uno de los huevos.

La escala de evaluación visual se compuso de diez categorías con valores enteros de cero a diez, donde el diez representó la mejor calidad interna (Figura 2). Para la puntuación de los huevos se utilizaron las imágenes de vista frontal y superior de cada huevo, cada una de las cuales fueron clasificadas en una de cinco categorías principales de la escala, representadas por los puntos pares $(2,4,6,8$ y 10$)$ las cuales describen cambios estructurales visibles en características físicas tanto de la albúmina como de la yema. Para cada puntaje se definieron las siguientes características:

10 Puntos: En vista superior se evidencian las fracciones densa y fluida de albumina, además la yema se encuentra centrada en la fracción densa. En la vista lateral se diferencia albumen denso del fluido, la yema sobresale del albumen y su forma es convexa.

8 puntos: En vista superior se evidencian las dos fracciones de la albúmina, siendo menos marcada su diferenciación. En la vista lateral no hay presencia de albumen denso, por su parte, la yema sobresale del albumen y mantiene la forma convexa. 
6 puntos: En la vista superior la fracción densa del albumen es difusa, la yema aumenta de diámetro y pierde su ubicación en el centro del albumen denso y comienza a desplazarse hacia los laterales. En la visión lateral no se diferencian las fracciones de la albumina y la yema pierde altura respecto al albumen y se observa levemente achatada.

4 puntos: En la vista superior no se diferencian las fracciones de la albumina, respecto a la yema se evidencia un aumento marcado del diámetro. En la visión lateral el nivel de la yema se presenta de forma plana, su altura es baja sin sobresalir del albumen.

\section{2 puntos: Rotura de yema.}

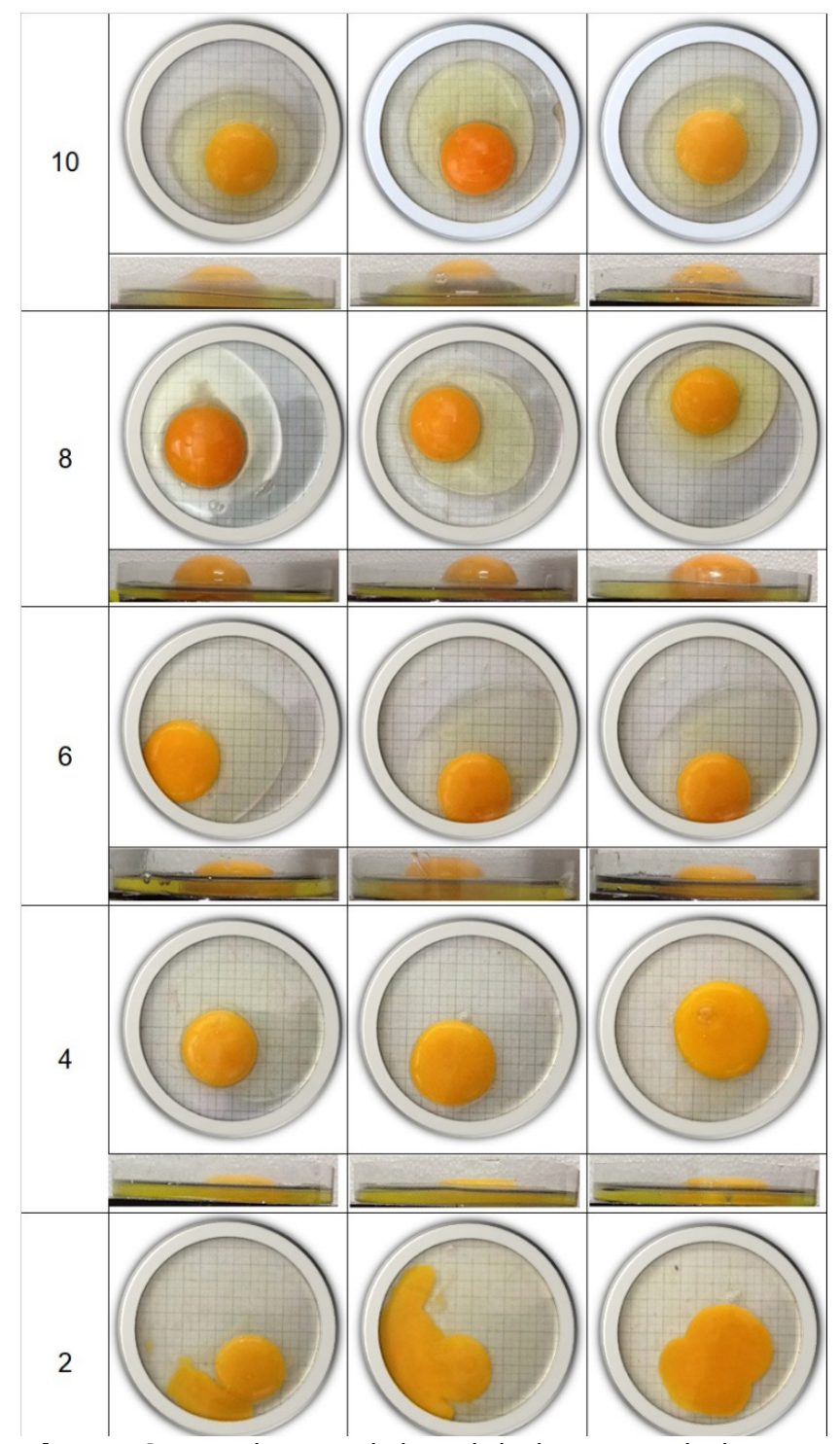

Figura 2. Escala visual de calidad interna de huevo. Categorías principales de la escala visual donde se pueden observar en cada una de las categorías tres imágenes de las vistas frontal y superior que ejemplifican las caracteristicas físicas descritas para cada una de las categorías
Finalmente, la puntuación de cada una de las dos fotografías de cada huevo fue promediada para obtener la puntuación de escala visual promedio, que representa el estado de calidad interna de cada huevo. En este procedimiento, se obtienen huevos clasificados en las puntuaciones intermedias representadas por las categorías impares de la escala $(3,5,7$ y 9$)$ que constituyeron puntos de transición entre las categorías principales de la escala en que los cambios fisicoquímicos del huevo pueden ser apreciados en solo una de las dos vistas del huevo.

Análisis estadístico. El presente estudio fue de tipo observacional retrospectivo. Los datos se analizaron utilizando el paquete estadístico licenciado STATA 16. Los datos de cada una de las variables fueron tabulados para obtener estadísticos descriptivos. Se utilizaron las pruebas de ANOVA y TUKEY para comparar las medias de cada variable de peso y calidad interna entre los diferentes tiempos de medición. En el caso de la escala visual por ser un variable de tipo categórica se describió utilizando la mediana y la moda en cada uno de los tiempos. Las correlaciones entre las variables de calidad interna (índice de yema, $\mathrm{pH}$ de yema, $\mathrm{pH}$ de albumen y escala visual) se analizaron mediante el procedimiento de coeficiente de correlación de Pearson y Spearman en el caso de la escala visual, además se determinó su asociación mediante modelo regresión lineal (Ec.1), previa comprobación de los supuestos y se realizó comprobación de fiabilidad del modelo.

\section{Ec.1. Modelo de regresión lineal}

Escala visual $=\mathrm{B} 0+\mathrm{B} 1$ (Indice de yema) $+\mathrm{B} 2(\mathrm{pH}$ de albumen) $+B 3(\mathrm{pH}$ de yema+B4 (mes de medicion $)+\varepsilon$

\section{RESULTADOS}

Peso de componentes del huevo. Al analizar el efecto del tiempo de almacenamiento a temperatura ambiente sobre el peso de los componentes del huevo, se observó una disminución estadísticamente significativa $(p<0.05)$ tanto en el peso absoluto como en el peso relativo de la albumina, la cual tuvo una reducción cercana al $10 \%$ al día 17 respecto a su peso absoluto inicial. Por su parte el tiempo de almacenamiento en anaquel no afectó el peso absoluto de la cáscara ni de la yema, mientras que respecto al peso relativo de la yema se observo un aumento $(p<0.01)$ significativo, además de 
Ángel-Isaza et al - Evaluación de escala visual de calidad interna de huevo

una mayor proporción yema: albumen al día 17 de almacenamiento respecto al día inicial de medición (Tabla 1). No se observaron diferencias significativas $(p>0.05)$ respecto al origen del huevo ni en el mes en que se realizó la medición.

Tabla 1. Peso de componentes de huevo de gallina ponedora comercial y su variación durante el tiempo de almacenamiento a temperatura ambiente.

\begin{tabular}{ccccccccc}
\hline \multirow{2}{*}{$\begin{array}{c}\text { Tiempo } \\
\text { (días) }\end{array}$} & Huevo & Cascara & Albumina & Yema & Cascara & Albumina & Yema & $\begin{array}{c}\text { Yema: } \\
\text { albumen }\end{array}$ \\
\cline { 2 - 9 }$y$ & $61.13 \mathrm{~b}$ & 7.32 & $38.46 \mathrm{c}$ & 15.35 & 11.98 & $62.91 \mathrm{~b}$ & $25.02 \mathrm{a}$ & $39.77 \mathrm{a}$ \\
6 & $60.90 \mathrm{~b}$ & 7.39 & $37.38 \mathrm{ab}$ & 16.12 & 12.17 & $61.32 \mathrm{~b}$ & $26.53 \mathrm{~b}$ & $43.26 \mathrm{ab}$ \\
9 & $60.23 \mathrm{ab}$ & 7.16 & $36.95 \mathrm{abc}$ & 16.15 & 11.88 & $61.31 \mathrm{ab}$ & $26.80 \mathrm{~b}$ & $43.71 \mathrm{ab}$ \\
13 & $58.22 \mathrm{ab}$ & 7.13 & $35.59 \mathrm{ab}$ & 15.49 & 12.28 & $61.13 \mathrm{ab}$ & $26.49 \mathrm{~b}$ & $43.33 \mathrm{ab}$ \\
17 & $57.37 \mathrm{a}$ & 7.01 & $34.63 \mathrm{a}$ & 15.65 & 12.25 & $60.36 \mathrm{a}$ & $27.27 \mathrm{~b}$ & $45.17 \mathrm{~b}$ \\
SEM & 0.34 & 0.05 & 0.23 & 0.13 & 0.01 & 0.01 & 0.04 & 0.34 \\
p valor & 0.002 & 0.077 & 0.000 & 0.417 & 0.082 & 0.000 & 0.000 & 0.004 \\
\hline
\end{tabular}

Letras $a, b$ y $c$ para diferencias significativas $(p<0.05)$ entre tiempos determinado pro pruebas de ANOVA y TUKEY. $n=105$ huevos por día de medición.

Calidad interna del huevo. Respecto a las variables medidas como marcadores de calidad interna del huevo, se observó un efecto significativo $(p<0.05)$ del tiempo de almacenamiento a temperatura ambiente sobre las variables de calidad interna de índice de yema, $\mathrm{pH}$ de albumen y $\mathrm{pH}$ de yema (Tabla 2 ), Al analizar la evolución del $\mathrm{pH}$ de los principales componentes internos del huevo en el tiempo de almacenamiento, se observó que tanto en el albumen como en la yema el pH aumentó. De igual forma el índice de yema presentó disminución significativa $(p<0.05)$ desde los primeros días de almacenamiento de los huevos.

Tabla 2. Variables de calidad interna de huevo de gallina ponedora comercial y su evolución en el tiempo de almacenamiento a temperatura ambiente.

\begin{tabular}{cccc}
\hline Tiempo & pH yema & pH albumen Índice de yema \\
\hline 3 & $6.10 \mathrm{a}$ & $8.91 \mathrm{a}$ & $0.42 \mathrm{a}$ \\
6 & $6.09 \mathrm{a}$ & $9.19 \mathrm{~b}$ & $0.34 \mathrm{~b}$ \\
9 & $6.11 \mathrm{a}$ & $9.20 \mathrm{~b}$ & $0.32 \mathrm{c}$ \\
13 & $6.21 \mathrm{~b}$ & $9.32 \mathrm{bc}$ & $0.31 \mathrm{C}$ \\
17 & $6.25 \mathrm{~b}$ & $9.38 \mathrm{c}$ & $0.26 \mathrm{~d}$ \\
SEM & 0.01 & 0.01 & 0.00 \\
P valor & 0.001 & 0.001 & 0.001 \\
\hline
\end{tabular}

Letras $a, b, c$ y $d$ para diferencias significativas $(p<0.05)$ entre tiempos determinado por pruebas de ANOVA y TUKEY $\mathrm{n}=105$ huevos por día de medición.

Al realizar la comparación de las puntuaciones de los huevos en la escala de evaluación visual entre los diferentes días se observó disminución estadísticamente significativa $(p<0.05)$ entre todos los días evaluados con excepción de los días 9 y 13 en cuyo caso no hubo diferencias. Como se observa en la tabla 3 al tercer día de postura la mayor cantidad de puntuaciones de escala visual se encuentran entre los nueve y diez puntos alcanzando al día 17 una moda y una mediana de cuatro puntos, puntuación que representa en características físicas el aumento en la fluidez del albumen que es percibido como un huevo de baja calidad (Tabla 3)

Tabla 3. Comportamiento de puntuaciones de escala de evaluación visual de huevo de gallina ponedora comercial durante el tiempo de almacenamiento a temperatura ambiente

\begin{tabular}{|c|c|c|c|c|c|c|c|}
\hline \multirow[t]{2}{*}{ Día } & \multicolumn{2}{|c|}{$\begin{array}{l}\text { Escala visual } \\
\text { promedio }\end{array}$} & \multicolumn{2}{|c|}{$\begin{array}{l}\text { Puntuación } \\
\text { vista superior }\end{array}$} & \multicolumn{2}{|c|}{$\begin{array}{l}\text { Puntuación } \\
\text { vista frontal }\end{array}$} & \multirow[t]{2}{*}{ CV* } \\
\hline & Me & $\mathbf{M}$ & Me & $\mathbf{M}$ & Me & $\mathbf{M}$ & \\
\hline 3 & 8 & 10 & 8 & 10 & 8 & 8 & 0.07 \\
\hline 6 & 6 & 6 & 6 & 6 & 6 & 6 & 0.04 \\
\hline 9 & 5 & 5 & 6 & 4 & 4 & 6 & 0.05 \\
\hline 13 & 5 & 5 & 6 & 4 & 4 & 6 & 0.04 \\
\hline 17 & 4 & 4 & 4 & 4 & 4 & 4 & 0.02 \\
\hline
\end{tabular}

$\mathrm{n}=105$ huevos por día de medición; $\mathrm{Me}=$ Mediana; $\mathrm{M}=$ Moda; $C V *$ Coeficiente de variación entre la puntuación en la escala visual promedio de los tres evaluadores para cada uno de los días de evaluación.

Correlación entre variables de calidad interna de huevo. En la tabla 4 se halla el resumen de resultados de las pruebas de 
correlación y regresión lineal entre las variables. Al realizar el análisis de correlación de Spearman entre las diferentes variables de calidad interna de huevo se observó correlación negativa entre el $\mathrm{pH}$ de la albumina con las variables de índice de yema y escala visual, Por su parte la correlación entre el índice de yema y escala visual fue positiva con un índice de correlación de 0.84 siendo estadísticamente significativa $(p<0.01)$, Respecto al $\mathrm{pH}$ de la yema se encontraron coeficientes de correlación más bajos que no fueron estadísticamente significativos ( $p>0.05)$.

Tabla 4. Coeficiente de correlación entre variable de calidad interna de huevo.

\begin{tabular}{ccccc}
\hline $\begin{array}{c}\text { Variable de } \\
\text { calidad }\end{array}$ & $\begin{array}{c}\text { Escala } \\
\text { visual }\end{array}$ & $\begin{array}{c}\text { Índice de } \\
\text { yema }\end{array}$ & $\begin{array}{c}\text { pH } \\
\text { albumen }\end{array}$ & pH yema \\
\hline $\begin{array}{c}\text { Escala visual } \\
\text { Índice de }\end{array}$ & 1 & & & \\
$\begin{array}{c}\text { yema } \\
\text { pH albumen }\end{array}$ & $-0.84^{* *}$ & 1 & & \\
pH yema & $-0.44^{*}$ & $-0.51^{*}$ & 1 & \\
\hline
\end{tabular}

Valores de coeficiente de correlación

Fiabilidad del modelo $\left(\mathrm{R}^{2}=0.74\right)$; Determinación de asociación mediante regresión lineal $\left(R^{2}=0.73\right)$.

$P$ valor de la asociación en el modelo de regresión lineal entre parejas de variables $* \mathrm{p}<0.05, * * \mathrm{p}<0.01$.

Al realizar la determinación de los intervalos de confianza (95\%) de las variables índice de yema y $\mathrm{pH}$ de albumen para los principales valores en la escala de evaluación visual de calidad de huevo (Tabla 5) se observaron rangos en índice de yema de 0.42 a 0.44 en la puntuación diez de la escala, mientras que cuanto la puntuación fue de cuatro, categoría en la cual las características físicas del huevo describen la pérdida de fluidez de la clara el índice de yema desciende a valores de entre 0.24 a 0.26 mientras que el $\mathrm{pH}$ del albumen aumento alcanzando el rango de entre 9.39 a 9.49 .

Tabla 5. Media e Intervalos de confianza del $95 \%$ de valores de índice de yema y $\mathrm{pH}$ de albumen en cada grado de escala visual.

\begin{tabular}{ccc}
\hline Escala visual & Índice de yema & pH de albumen \\
\hline 4 & $0.25(0.24-0.26)$ & $9.44(9.39-9.49)$ \\
6 & $0.33(0.32-0.35)$ & $9.24(9.19-9.30)$ \\
8 & $0.41(0.39-0.44$ & $9.07(9.01-9.19)$ \\
10 & $0.42(0.41-0.44)$ & $8.92(8.75-9.09)$ \\
\hline
\end{tabular}

\section{DISCUSIÓN}

Escala de evaluación visual. En la metodología de Van y Wilgus (6) en 1935 los autores asignaron una escala visual con valores que van desde 1 hasta 5 con intervalos de 0.5 , utilizando tanto la vista frontal y superior, y describiendo la posición y distribución alrededor de la yema, siendo el 5 el puntaje más bajo donde no se observan diferencias entre las dos fracciones de albúmina.

Con base a los estudios previos de Wagenen y Wilgus (6) y Sastre et al (5) se desarrolló la escala de evaluación visual utilizada en la presente investigación, la cual se relacionó con otras variables indicadores de calidad interna como índice de yema, encontrándose que con índices de yema superiores a 0.38 los huevos se encuentran en categorías superiores de la escala de evaluación visual ( $>8$ ), mientras que cuando en puntuaciones indicativas de calidad pobre $(<5)$ el índice de yema adoptó valores inferiores a 0.26 , de esta manera se observó que la escala de evaluación visual empleada en la investigación permitió evidenciar cualitativamente cambios físico químicos que ocurrieron en los componentes del huevo, relacionados con su calidad y frescura. Por su parte se hace necesario para futuras investigaciones evaluar su relación con otras mediadas de calidad de huevo como las UH además de la influencia de la edad de las aves en los diferentes índices de calidad incluyendo la escala de medición visual.

Respecto a la variación entre las puntuaciones realizadas por los tres evaluadores de los huevos dentro de la escala visual promedio, se hallaron coeficientes de variación entre 0.08 y 0.03 , encontrándose así pequeñas diferencias en las puntuaciones de algunos huevos entre los evaluadores. Estas discrepancias dadas por a la subjetividad de una evaluación visual se hacen menos pronunciada en los últimos días de evaluación donde hay mayor cantidad de huevos puntuados entre las categorías 2 y 4 las cuales presentan características visuales más fácilmente distinguibles como lo son la pérdida total del albumen denso (categoría 4) y la rotura de la yema (categoría 2 ). No se produjeron diferencias de más de dos puntos en la evaluación del mismo huevo por parte de los diferentes expertos, por lo que el coeficiente de variación se mantuvo en niveles bajos durante toda la prueba. 
Peso de componentes del huevo y Calidad interna del huevo. En el presente estudio se observó la pérdida de peso absoluto y relativo del albumen durante el tiempo de almacenamiento, lo cual tiene relación con lo observado por Pissinati et al (7) en 2014, quienes reportan una pérdida cercana a dos puntos porcentuales en el peso relativo del albumen en un periodo de 21 días de almacenamiento a temperatura ambiente que tuvo un promedio de $25^{\circ} \mathrm{C}$. Por su parte lo observado por Akter et al (8) en 2014 muestra una pérdida significativa $(p<0.01)$ en el peso relativo del albumen a los 21 días de almacenamiento a $28^{\circ} \mathrm{C}$, de cuatro puntos porcentuales, siendo una reducción mayor a la encontrada en el presente estudio. En el trabajo realizado por Jin et al (9) en 2011 expusieron huevos a diferentes temperaturas de almacenamiento $\left(5,21\right.$ y $29^{\circ} \mathrm{C}$ ) hallaron que tanto el aumento de la temperatura como el tiempo de almacenamiento afectaron negativamente peso de la albumina.

Akter et al (8) en 2014 reportaron que la pérdida de peso del huevo depende de la temperatura, encontrando que los huevos almacenados a $4^{\circ} \mathrm{C}$ mostraron una pérdida de peso significativamente $(p<0.05)$ menor que a temperatura ambiente, explicando que esto, puede deberse a la menor pérdida de agua y otros productos gaseosos del contenido del huevo cuando la temperatura ambiental es menor (8). La pérdida de humedad y gases del interior por los poros de la cascara del huevo comienza desde el momento de la postura y se exacerba con el tiempo de almacenamiento y algunos factores además de la temperatura ya mencionada donde se encuentran la humedad relativa y el flujo de aire al que se sometan los huevos durante el almacenamiento y que determinan el grado de pérdida de peso que presenta el huevo y especialmente la albumina (10).

En el presente estudio se observó que el pH de la albumina presento un crecimiento significativo ( $p$ $>0.05$ ) durante el tiempo de almacenamiento, siendo esto congruente con los resultados de otros autores que llevaron a cabo mediciones similares $(11,12)$. Como ha sido reportado el $\mathrm{pH}$ de la albumina actúa como un indicador de la frescura del huevo debido a su relación con los procesos químicos que ocurren en su interior (7), donde los huevos expuestos al ambiente presentan un intercambio de gases en el que el huevo pierde $\mathrm{CO} 2$ que pasa a través de los poros de la cáscara proveniente de la disociación del ácido carbónico que es parte del sistema buffer de la albúmina (1)

Por su parte, estos cambios químicos ocurridos por el intercambio de gases en el interior del huevo que se ven reflejados en el aumento del $\mathrm{pH}$, se encuentran además relacionados con la calidad sensorial del huevo y las características del albumen. La entrada de oxígeno en el huevo provoca un aumento en el tamaño de la cámara de aire $(1,10)$, por su parte el aumento en el pH de la albúmina conduce a la pérdida de complejo proteicos de manera que el albumen pierde viscosidad y con ello la perdida de la fracción densa de la albumina $(1,10)$.

Estas reacciones químicas con consecuencias negativas sobre la calidad interna del huevo y sobre características sensoriales como es la viscosidad de la albúmina y la presencia de una capa densa de albumina que rodea la yema, pudo ser evidenciado y medido por medio de la escala visual empleado en el presente estudio, donde la puntuación promedio de la escala visual disminuyó con el tiempo del almacenamiento del huevo al igual que el índice de yema, encontrándose a su vez una correlación significativa $(p<0.05)$ de $0.84\left(R^{2}=0.73\right)$.

En conclusión, en este estudio se observó que el tiempo de almacenamiento a temperatura de 29 a $32^{\circ} \mathrm{C}$ afecta el peso relativo del albumen y los parámetros de calidad interna de huevo tales como el índice de yema y el pH de la albumina, además de disminuyo la puntuación de la escala de evaluación visual. La escala visual empleada permitió identificar cambios físicos observables en los componentes internos del huevo (yema y clara) y puntuarlos en una escala para identificar el grado de degradación. Se debe continuar evaluando el comportamiento de la escala frente a otras variables de calidad internas de huevo.

\section{Conflicto de intereses}

Los autores declaran que no tienen ningún conflicto de intereses en relación con este trabajo. 


\section{REFERENCIAS}

1. Hagan JK, Eichie FO. Egg quality of two layer strains as influenced by extended storage periods and storage temperatures. Livest Res Rural Dev. 2019; 31(9). http://www. Irrd.org/Irrd31/9/jhagan31145.html

2. Marzec A, Damaziak K, Kowalska H, Riedel J, Michalczuk M, Koczywąs E, et al. Effect of Hens Age and Storage Time on Functional and Physiochemical Properties of Eggs. J Appl Poult Res. 2019; 28(2):290-300 https://doi.org/10.3382/japr/pfy069

3. Dong X, Dong J, Peng Y, Tang X. Comparative study of albumen $\mathrm{pH}$ and whole egg $\mathrm{pH}$ for the evaluation of egg freshness. Spectrosc Lett. 2017; 50(9):463-469. https://doi.org L10.1080/00387010.2017.1360357

4. Chung $\mathrm{SH}$, Lee KW. Effect of hen age, storage duration and temperature on egg quality in laying hens. Int J Poult Sci. 2014; 13(11):634-636. https://doi.org/10.3923/ ijps.2014.634.636

5. Sastre A, Sastre R, Tortuero F, Suarez G, Vergara G, López C. Lecciones sobre el huevo. $1^{\text {a }}$ ed. Madrid: Instituto de Estudios del Huevo; 2002. http://www.institutohuevo. com/wp content/uploads/2017/07/ Lecciones-del-huevo-completo.pdf

6. Van A, Wilgus HS. The determination and importance of the condition of the firm albumen in studies of egg white quality. Jour Agr Res. 1935; 51(1):1129. https://naldc. nal.usda.gov/download/IND43968622/PDF

7. Pissinati A, Oba A, Yamashita F, Da Silva CA, Pinheiro JW, Roman JMM. Qualidade interna de ovos submetidos a diferentes tipos de revestimento e armazenados por 35 dias a $25^{\circ} \mathrm{C}$. Semin Cienc Agrar. 2014; 35(1):531540. http://doi.org/10.5433/16790359.2014v35n1p531
8. Akter $Y$, Kasim A, Omar H, Sazili AQ. Effect of storage time and temperature on the quality characteristics of chicken eggs. J Food Agric Environ. 2014; 12(2):87-92. http://citeseerx.ist.psu.edu/viewdoc/ summary?doi=10.1.1.654.3052

9. Jin $\mathrm{YH}$, Lee KT, Lee WI, Han YK. Effects of storage temperature and time on the quality of eggs from laying hens at peak production. Asian-Australas J Anim Sci. 2011; 24(2):279-284. https://doi. org/10.5713/ajas.2011.10210

10. Feddern V, Prá $M C D$, Mores $R$, Nicoloso RDS, Coldebella A, Abreu PGD. Egg quality assessment at different storage conditions, seasons and laying hen strains. Ciênc agrotec. 2017; 41(3):322-333. https://doi. org/10.1590/1413-70542017413002317

11. Arruda MD, Gouveia JWF, Lisboa ACC, de Lima Abreu AC, de Abreu AKF. Avaliação da qualidade de ovos armazenados em diferentes temperaturas. Revista Craibeiras de Agroecologia. 2019; 4(1):7681. http://200.17.114.107/index.php/era/ article/view/7681

12. Lee $M H$, Cho EJ, Choi ES, Sohn SH. The effect of storage period and temperature on egg quality in commercial eggs. Korean J Poult Sci. 2016; 43(1):31-38. http://dx.doi. org/10.5536/KJPS.2016.43.1.31 